\title{
MP8 MONOCLONAL GAMMOPATHY CHARACTERIZATION USING SERUM PROTEIN ELECTROPHORESIS IN A MAJOR URBAN POPULATION
}

DB Laskar, K Shafique, C Lu, A Zuretti. Pathology, SUNY Downstate Medical Center, Brooklyn, NY, United States

\subsection{6/jim-2016-000080.20}

Purpose of Study Serum protein electrophoresis (SPEP) with subsequent immunofixation (IF) are clinical laboratory techniques used to evaluate a wide-range of disorders where abnormal serum protein quantities are characteristic (e.g. multiple myeloma (MM), MGUS, amyloidosis, HIV/ AIDS, SLE, CLL/NHL). Thus, it is important to identify or exclude malignancy when considering the analyses. Our aim is to characterize SPEP patients from our institution, a predominantly black population.

Methods Used We retrospectively reviewed 50 patient's SPEP/IF results. Data recorded were SPEP/IF results, monoclonal immunoglobulin (Ig) identity, clinical diagnoses, age, race and gender. Univariate analysis was used to describe patient demographics. Parametric analysis was used to compare the monoclonal gammopathy (MG) group versus non-MG group.

Summary of Results Age range was $12-86$ years, mean age was 62 years and male to female ratio was 1:3.5. Forty-eight (96\%) patients identify as black, 1 (2\%) Asian 


\section{Abstracts}

Table 1. Patient mean age gender with corresponding SPEP result.
\begin{tabular}{|c|c|c|c|c|c|c|}
\hline & $\begin{array}{c}\text { Acute } \\
\text { Inflammation }\end{array}$ & $\begin{array}{c}\text { Chronic } \\
\text { Inflammation }\end{array}$ & Inconclusive & $\begin{array}{c}\text { Monoclonal } \\
\text { band }\end{array}$ & Normal & $\begin{array}{c}\text { Polyclonal } \\
\text { band }\end{array}$ \\
\hline No. patients & $1(2 \%)$ & $3(6 \%)$ & $24(48 \%)$ & $16(32 \%)$ & $3(6 \%)$ & $3(6 \%)$ \\
\hline Age (mean) & 37 & 48 & 59 & 69 & 69 & 58 \\
\hline Gender (M:F) & $0: 1$ & $1: 2$ & $1: 3$ & $1: 4$ & $0: 1$ & $1: 2$ \\
\hline
\end{tabular}

\section{Abstract MP8 Figure 1}

and $1(2 \%)$ white. SPEP patterns showed $1(2 \%)$ patient had acute inflammation, $3(6 \%)$ had chronic inflammation, $24(48 \%)$ were inconclusive, 16 (32\%) had MGs, 3 (6\%) had normal results and $3(6 \%)$ had polyclonal bands (table 1). Among MG patients, IgG was most common isotype (75\%), kappa was most common light chain (58\%) and IgG kappa was most common (44\%). Mean age was 69 years for MG patients and 58 years for non-MG patients.

MM was identified in $9(18 \%)$ patients; 89\% (8/9) had normal total protein (TP) levels and $1(11 \%)$ had increased TP. Neuropathy was seen in 7 (14\%) patients; 71\% (5/7) had polyclonal gamma Ig increase, and $1(14 \%)$ case with co-HIV infection had monoclonal IgG kappa. Seven (14\%) patients had CKD, $4(8 \%)$ had HIV/AIDS, $3(6 \%)$ had anemia, $3(6 \%)$ had MGUS, $1(2 \%)$ had SLE and the remaining 16 (32\%) had other co-morbidities (i.e. HTN, $\mathrm{DM}, \mathrm{CAD}$, etc.).

Conclusions SPEP/IF analyses were used to characterize 50 patients. A wide-range of disorders were observed. MG patients were 11 years older than non-MG patients. IgG kappa was most common MG. Our study showed femalepredominance. This study shows SPEP utility to discern various disorders observed at our institution. 